\title{
Extended medial sural artery perforator free flap for groin and scrotal reconstruction
}

\author{
Chad M. Teven ${ }^{1}$, Jason W. Yu², Lee C. Zhao ${ }^{3}$, Jamie P. Levine ${ }^{2}$ \\ ${ }^{1}$ Division of Plastic and Reconstructive Surgery, Mayo Clinic, Phoenix, AZ; ${ }^{2}$ Hansjörg Wyss Department of Plastic Surgery and ${ }^{3}$ Department \\ of Urology, NYU Langone Health, New York, NY, USA
}

The medial sural artery perforator (MSAP) flap is a versatile fasciocutaneous flap that has been used successfully in the reconstruction of defects across the body. In specific cases, it may prove superior to more commonly used options (e.g., anterolateral thigh flap and radial forearm free flap). Historically, a disadvantage of the MSAP flap is the relatively small surface area it provides for reconstruction. We recently encountered a patient with extensive pelvic injuries from prior trauma resulting in significant scarring and contracture of the groin, tethering of the penis, and loss of the scrotum and one testicle. The patient was unable to achieve erection from tethering and his remaining testicle had been buried in the thigh. In considering the reconstructive options, he was not a suitable candidate for a thigh-based or forearmbased flap. An extended MSAP flap measuring $25 \mathrm{~cm} \times 10 \mathrm{~cm}$ was used for resurfacing of the groin and pelvis as well as for the formation of a neoscrotum. This report is the first to document an MSAP flap utilized for simultaneous groin resurfacing and scrotoplasty. Additionally, the dimensions of this flap make it the largest recorded MSAP flap to date.

Keywords Extended medial sural artery perforator flap / Groin reconstruction / Medial sural artery perforator / Scrotal reconstruction / Scrotoplasty
Correspondence: Chad M. Teven Division of Plastic and Reconstructive Surgery, Mayo Clinic, 5779 E Mayo Blvd, Phoenix, AZ 85054, USA

Tel: +1-480-342-2000

Fax: +1-480-764-1002

E-mail: Teven.chad@mayo.edu

Received: December 29, $2019 \bullet$ Revised: January 30, $2020 \bullet$ Accepted: February 5, 2020

pISSN: 2234-6163 • elSSN: 2234-6171 • https://doi.org/10.5999/aps.2019.01921 • Arch Plast Surg 2020;47:354-359

\section{INTRODUCTION}

The concept of a flap utilizing medial calf tissue and based on the medial sural vessels was first introduced by Taylor and Daniel in 1975 [1]. Several decades would pass, however, before Montegut and Allen [2], Cavadas et al. [3], and others applied such flaps clinically, eventually refining them to perforator-based flaps that spared the underlying gastrocnemius muscle. Many authors would now consider the medial sural artery perforator (MSAP) flap an excellent option for numerous reconstructive challenges [4]. The MSAP flap is a relatively thin and pliable fasciocutaneous flap with a long vascular pedicle and vessels of adequate caliber for microsurgical anastomosis. Its dissection as a perforator flap preserves the structure and function of the underlying gastrocnemius muscle. It can also be harvested as a chimeric flap to include muscle, tendon and/or nerve if desired. It has been successfully used for numerous applications, including reconstruction of the head and neck [5], hand and upper extremity [6], and lower extremity [7]. Indeed, it may be used in place of more conventional options such as the anterolateral thigh (ALT) flap and the radial forearm free flap (RFFF) depending on the reconstructive goals, defect and donor site characteristics, and patient desires. Disadvantages of the MSAP flap include its relatively small size, variable location and dimensions 
of perforating vessels, and an unacceptable donor site appearance for some patients [8].

In the current report, we highlight a novel application of an MSAP flap that has not been previously described. A patient who suffered extensive traumatic injuries to the groin, penis, and scrotum underwent reconstruction of the involved tissues with an extended MSAP free flap. A single large flap was used for resurfacing of the groin and penis as well as in the creation of a neoscrotum. A recently published systematic review of 35 studies involving the use of the MSAP flap reported average flap dimensions of $6.0 \pm 2.3 \mathrm{~cm} \times 9.8 \pm 3.6 \mathrm{~cm}$ [4]. The case reported herein utilized an MSAP flap that was $25 \mathrm{~cm} \times 10 \mathrm{~cm}$, which is the largest documented MSAP flap to date. In this paper, we share our experience with the use of an extended MSAP for groin and scrotal reconstruction and also review the indications, applications, and surgical anatomy of the MSAP flap. The patient provided written consent for use of information regarding his diagnosis/treatment and photographs.

\section{CASE}

A 31-year-old male patient suffered extensive traumatic injuries to the pelvis with significant loss of scrotal and penile tissue secondary to a work-related grinder accident (Fig. 1). He also suffered a concomitant injury to the left upper extremity requiring transhumeral amputation. Acute management at an outside hospital included resection of the scrotum and left testicle due

\section{Fig. 1. Preoperative photograph}

The patient suffered extensive traumatic damage to the pelvis and groin secondary to an occupational injury resulting in severe scarring and contracture to the pubis, groin, and thighs and loss of the scrotum and left testicle. The right testicle had been buried in the right thigh.

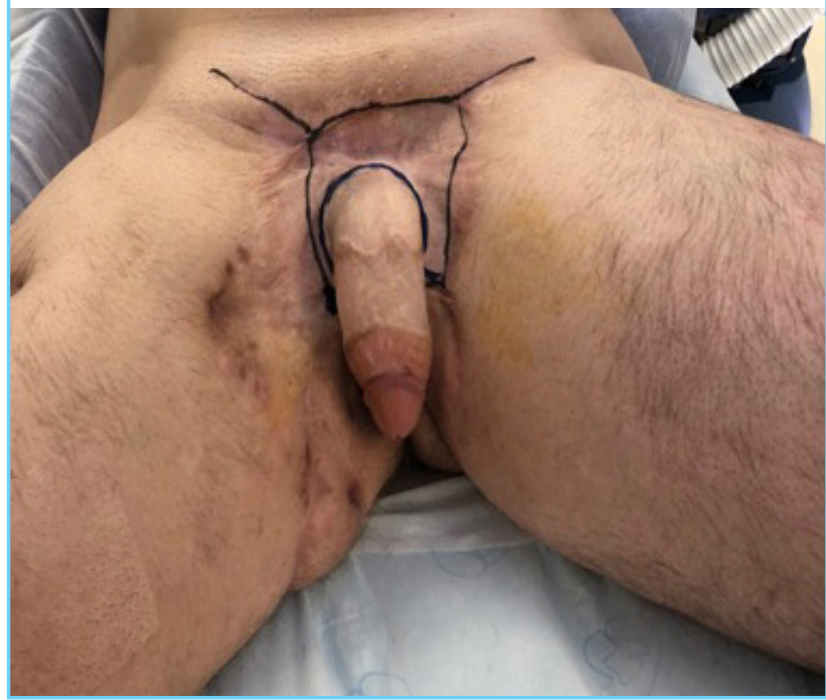

to injury, burial of the remaining testicle into the right thigh, and debridement of nonviable groin and penile soft tissue. He subsequently underwent split thickness skin grafting to the open wounds on his penis, thighs, and pelvis. One year later, he was referred to our institution for further management. Upon presentation, he reported significant pain and discomfort to the affected areas, an inability to achieve erection, and documented azoospermia. Examination demonstrated scarring and contracture of the penis and groin. Erection was mechanically hindered from significant tethering on the ventral penile surface. Azoospermia was thought to be due to burial of the testicle within the thigh, exposing it to supraphysiologic temperatures as Doppler ultrasound of the testicle demonstrated adequate perfusion.

Goals of reconstruction included removal and replacement of scarred and contracted tissue with healthy, vascularized tissue; creation of a sensate neoscrotum; and the ability to achieve erection. The patient was not an appropriate candidate for thighbased reconstruction due to excess adipose tissue and significant scarring of the thighs, nor forearm-based reconstruction due to patient refusal of using a forearm donor site given the loss of the contralateral arm. The decision was made to proceed with a large (i.e., extended) chimeric MSAP flap that included a sensory nerve for neurotization of the neoscrotum.

Initially, excisional and incisional scar release was performed from the pubic symphysis to the perineum and right medial thigh, resulting in significant wound development (Fig. 2). Further scar was released from the ventral and dorsal penile surfac-

\section{Fig. 2. Intraoperative photograph prior to reconstruction}

Scarring and contracture about the penis and groin were released and excised, resulting in large wounds in the pubic region, groin, and perineum.

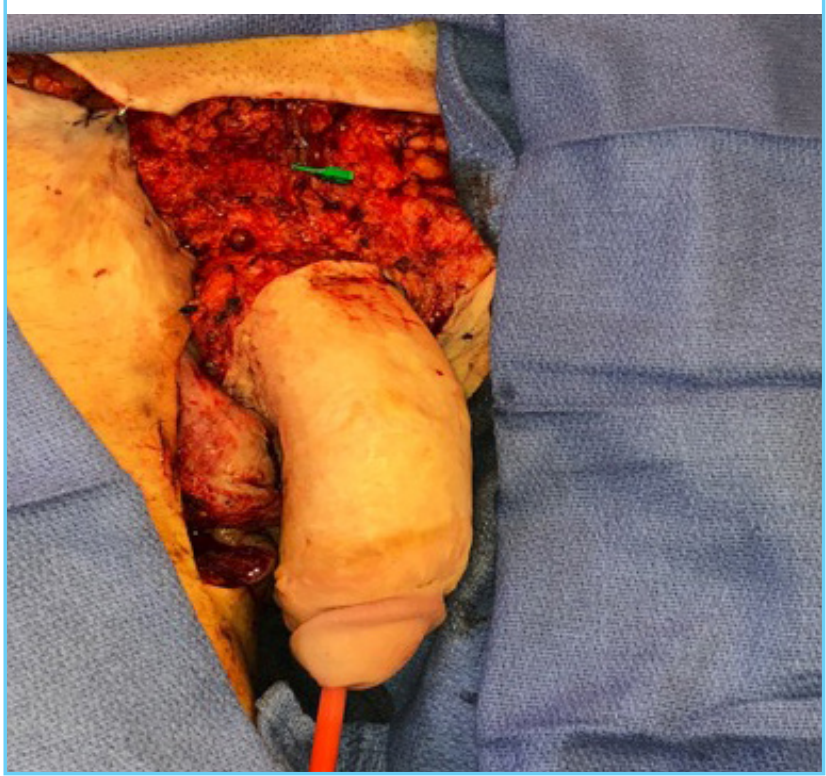


es, facilitating anterior advancement of the shaft. The left deep inferior epigastric vessels and the ilioinguinal nerve were exposed in preparation for free tissue transfer. The right testicle was mobilized from the right thigh for repositioning.

An extended left MSAP flap measuring $25 \times 10 \mathrm{~cm}$ was designed with its skin paddle overlying the presumed dominant perforator. Given the large size of the flap, three perforators from the medial sural pedicle were included in the harvest (Fig. 3). At

\section{Fig. 3. Medial sural artery perforator flap \\ A left chimeric medial sural artery perforator flap measuring 25 $\mathrm{cm} \times 10 \mathrm{~cm}$ and included three perforators, two veins, and the pos- terior femoral cutaneous nerve was harvested for resurfacing of the wounds and scrotal reconstruction.}

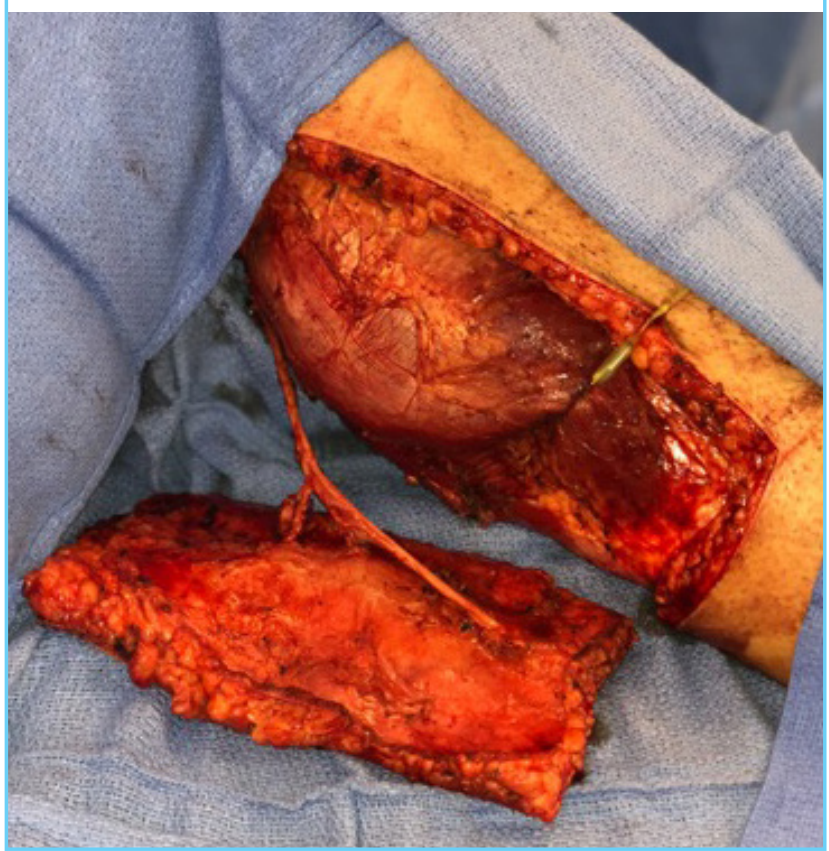

transfer, two veins were included in the anastomosis to ensure adequate drainage. The flap was elevated in chimeric fashion by including the posterior femoral cutaneous nerve, which would be coapted to the ilioinguinal nerve.

Upon transfer, the flap was initially anchored to the superior base of the penis to provide coverage to the wounds superior to the penis (Fig. 4A). In order to cover the wounds below the penis, a full-thickness vertical incision was made in the flap through which the penis was introduced (Fig. 4B). Care was taken to avoid injury to the perforators or pedicle. The inferior aspect of the flap was further designed into a neoscrotum, into which the testicle was secured (Fig. 4C). A split thickness skin graft $\left(200 \mathrm{~cm}^{2}\right)$ was required for donor site closure. The postoperative course was uncomplicated (Fig. 5A), and restrictions included avoiding bathing, lifting, pushing, and pulling for 6 weeks. Six months after surgery, the patient was fully healed, pain-free, and able to achieve erection (Fig. 5B).

\section{DISCUSSION}

The MSAP flap has become a versatile option in the reconstruction of various defects across the body. As a relatively thin and pliable fasciocutaneous flap, it can be used for many defects that have historically been reconstructed using an ALT flap or RFFF. In a 2019 systematic review and meta-analysis of the MSAP flap, Daar et al. [4] found that the most common indications for use of an MSAP flap are oncologic and traumatic defects. Other indications include infection, burn reconstruction, and salvage surgery. The most frequent recipient sites are the oral cavity and foot/ankle. Other common recipient sites include the head/ neck, hand/wrist, and lower extremity [4]. It may be used as a

\section{Fig. 4. Intraoperative photographs after flap transfer}

The flap was transferred and subsequently fashioned to achieve the goals of reconstruction. (A) Flap transferred and secured superiorly to the penis. (B) A longitudinal slit was created in the flap to allow the penis to be brought through. Care was taken to avoid injuring the perforating vessels. (C) The right testicle was secured in the inferior aspect of the flap that, which was used for construction of the neoscrotum.
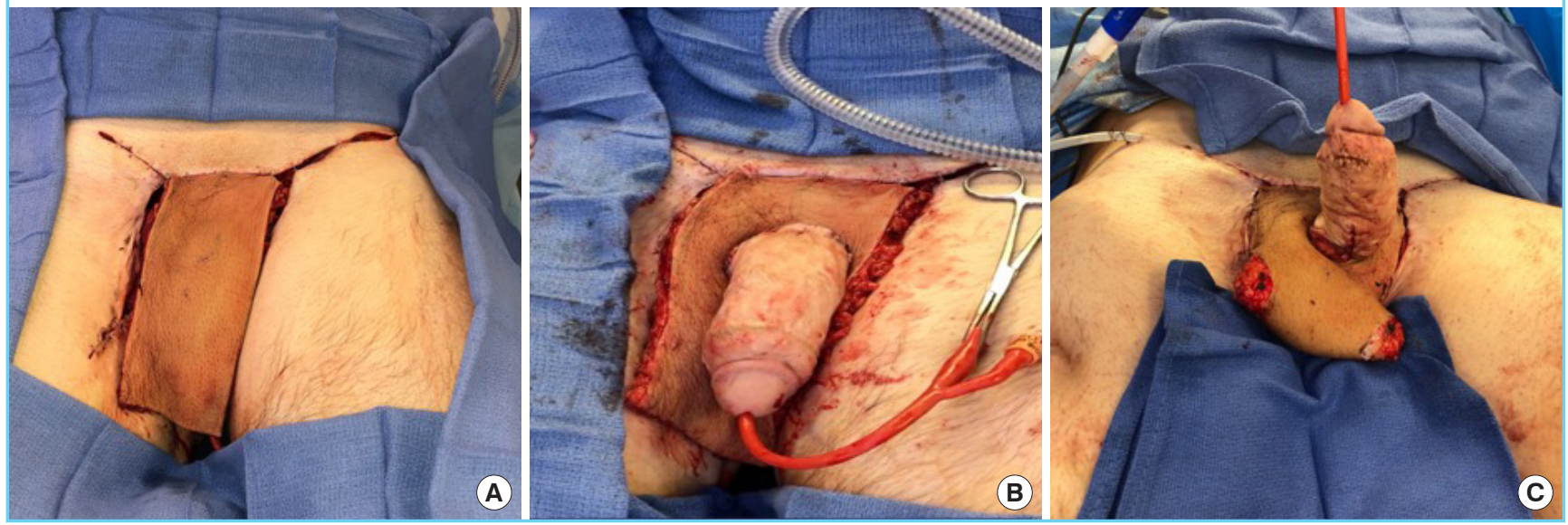


\section{Fig. 5. Postoperative photographs}

The flap was used for resurfacing of groin and perineal wounds and also to reconstruct the scrotum. (A) On table postoperative outcome. (B) Six-month follow-up.
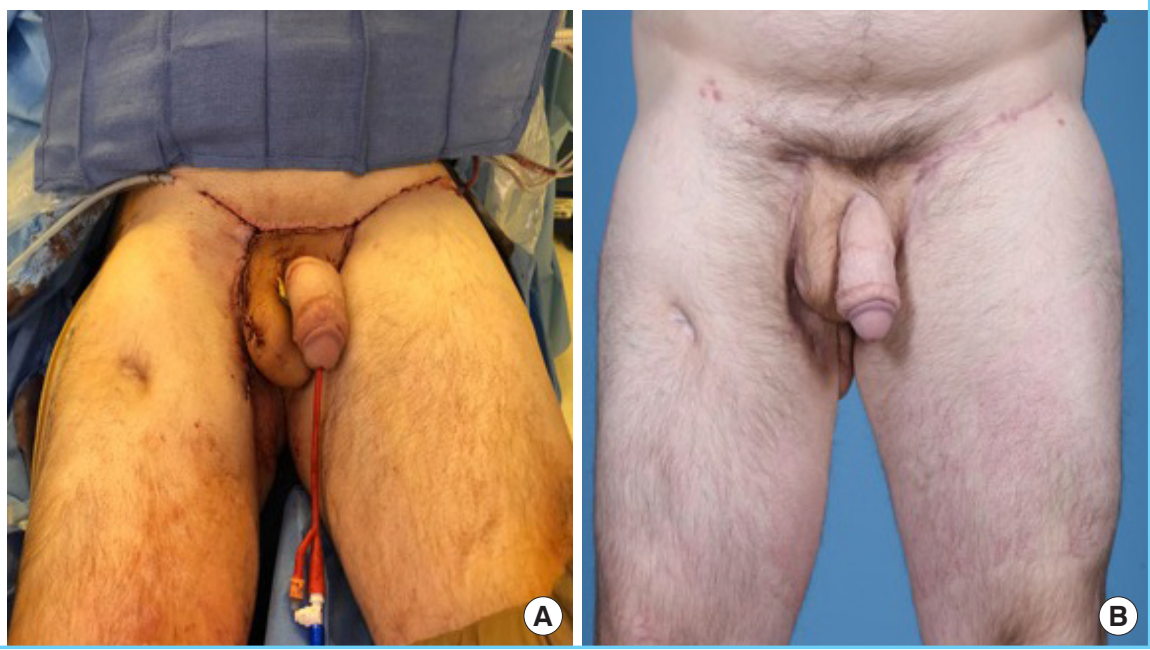

pedicled or free flap.

The MSAP flap is typically employed for small to mediumsize defects. In previous studies, the average flap length is $9.8 \mathrm{~cm}$ (range, 3-23 cm) and the average flap width is $6.0 \mathrm{~cm}$ (range, $2.5-17 \mathrm{~cm}$ ) [4]. The current case necessitated a large flap due to extensive damage to the pelvic region as well as the need for scrotoplasty. The flap dimensions were $25 \mathrm{~cm} \times 10 \mathrm{~cm}$, which is the largest reported MSAP in the literature. In addition, the pedicle length was $16 \mathrm{~cm}$, which, although not the longest reported, is significantly longer than the average pedicle length of $10.1 \mathrm{~cm}[4]$.

The medial sural artery arises from the popliteal artery in the distal popliteal fossa before entering the medial gastrocnemius muscle and follows a course roughly approximating a line drawn from the middle of the popliteal crease to the medial malleolus. Cavadas et al. [3] reported an average of 2.2 perforators (range, 1-4) piercing through the medial gastrocnemius muscle toward the overlying adipocutaneous tissue. The first perforator usually branches $8-10 \mathrm{~cm}$ from the popliteal crease and the most distal $16-18 \mathrm{~cm}$ from the crease [8]. If a sensate flap is desired, the posterior cutaneous nerve of the thigh or, as in the current case, the posterior femoral cutaneous nerve can be included. In addition, it may be harvested as a chimeric flap via inclusion of the gastrocnemius muscle or plantaris tendon [9].

During harvest, the patient can be positioned frog-legged or prone. A hand-held Doppler will aid in identifying the perforators. Elevation typically begins at the anterior border of the flap (medial side of the leg) and continues posteriorly until perforators are identified. Once a perforator(s) of adequate caliber is noted, it can be traced proximally toward its origin via intramuscular dissection through the gastrocnemius fibers. Thereafter, the remaining borders of the flap can be incised. The lesser sa- phenous vein can be included for augmented venous drainage. The motor nerve to the medial belly of the gastrocnemius may be encountered and should be preserved [10].

Although the MSAP flap has become an effective reconstructive option, one must be aware of its limitations. Historically, the MSAP flap was thought to be well-suited for small to mediumsized defects. Although the maximum size for this flap remains unknown, the flap reported herein challenges previous boundaries, extending across several nearby angiosomal territories of the posterior leg. These include the territories of the posterior tibial, peroneal, and lateral sural arteries, as confirmed by cadaveric studies by Taylor and Pan [11]. Furthermore, this flap demonstrates that the extended integument is not necessarily limited by the angiosomal territory of the source vessel, specifically the medial sural artery. Rather, the extent of "choke anastomoses" of adjacent territories are challenged given the immediate harvest of this large flap [12].

A recent study of MSAP reconstruction of limb defects reported the largest flap used in their series of 23 flaps was $14 \mathrm{~cm} \times 9$ $\mathrm{cm}$ [13]. Ours is the first study to report using a flap that is 25 $\mathrm{cm} \times 10 \mathrm{~cm}$. However, we had the benefit of including three adequately sized perforators. In cases without favorable vessel anatomy, harvesting a large flap may significantly increase the risk of total or partial necrosis. A further limitation is anatomic variability of the perforators with respect to location and caliber noted by some authors [4]. The use of perioperative imaging in the form of Doppler ultrasonography and computed tomographic angiography can improve the accuracy of perforator assessment [14]. Finally, dissection of the MSAP flap can be tedious due to small perforators with a long intramuscular course and therefore may be extremely challenging for inexperienced surgeons. 
A further consideration of the MSAP flap is potential donor site morbidity. Harvesting of even a small piece of tissue from the posterior lower leg will often result in a contour deformity. Flaps that are $>5 \mathrm{~cm}$ in width should close primarily. However, a consequence of the relatively high degree of tension on the closure at this location is widening of the scar [13]. Defects $>5$ $\mathrm{cm}$ require skin grafting of the donor site, which some patients may find unacceptable. Other than a potentially aesthetically displeasing appearance, MSAP donor site complications are rare and minor in nature. These consist of delayed healing, dehiscence, and altered sensation, and occur in $<2 \%$ of patients [4]. This rate compares favorably with ALT flap and RFFF reconstruction [15]. Furthermore, functional deficit of the donor site is minimal after MSAP harvest.

Although the complication rate associated with the use of MSAP flaps is comparable to the complication rates of other flaps used for similar indications [4], one must be cognizant of the potential for inadequate venous outflow as it is the most common cause of flap failure. In general, both venae comitantes (VC) of the medial sural artery are anastomosed to recipient veins. Although this is usually sufficient for venous drainage, it may not be if one of the VCs is of inadequate caliber and/or nondominant. Strategies to reduce complications related to venous outflow include the incorporation of a superficial vein (e.g., lesser saphenous vein) into the flap and external monitoring with a flow coupler [13].

The indications for use of an MSAP flap are similar to those for which an ALT flap or RFFF would be appropriate. In general, an ALT flap provides significantly more tissue than an MSAP flap can. Furthermore, the harvest of a fasciocutaneous or chimeric ALT flap is more straightforward than for a fasciocutaneous or chimeric MSAP flap. However, in many patients, particularly those who are obese, an ALT flap is too thick to provide for an adequate reconstruction. This is also true for the reconstruction of intraoral defects, where the bulkiness of an ALT flap may prove detrimental. Compared to a RFFF, a major benefit of an MSAP flap is the sparing of vital structures (e.g., radial artery). In addition, the MSAP flap donor site is far more inconspicuous than the forearm donor site. Tendon exposure may also plague the donor site after RFFF harvest. In contrast, the RFFF is more likely to offer thin and pliable tissue as well as demonstrate consistent anatomy.

In the current case report, the decision was made to use an MSAP flap for reconstruction for several reason. Although an ALT flap would have provided enough tissue, the patient was not an appropriate candidate for thigh-based reconstruction due to the thickness and previous scarring of his thighs. Similarly, he was deemed not to be an appropriate candidate for fore- arm-based reconstruction as a RFFF would have not provided a sufficient quantity of tissue and also because of his refusal to use the arm as a donor site given his previous amputation to the contralateral arm. Other options included tissue transfer from the abdomen or back. The decision was made to use an MSAP flap as it had the potential to provide an adequate amount of vascularized tissue and was pliable enough to contour appropriately. Given the occasionally inconsistent anatomy of the flap and because we were unsure of the size of the flap that would be needed to accomplish our reconstructive goals, the patient was counseled that a second flap may be needed if the first is insufficient. During the procedure, the favorable vascular anatomy allowed for an extremely large flap to be harvested as well as for unique tailoring of the flap to fit the defect. The goals of the procedure were to remove all scarred and contracted tissue from the affected sites, resurface the groin and pelvic wounds with healthy vascularized tissue, and create a neoscrotum into which the testicle would be secured. A large MSAP flap with three perforators was used to accomplish this. The patient subsequently reported reduced pain in the areas injured by the initial traumatic event and also a return in his ability to achieve erection. Due to the size of the MSAP flap, a large skin graft was required for coverage of his donor leg. The patient tolerated this and reported no negative consequences.

The current report illustrates a novel use of an MSAP flap in the treatment of a difficult problem. We report for the first time using an MSAP flap for groin and penile resurfacing and for creation of a neoscrotum. Furthermore, this was accomplished with the largest reported MSAP flap to date, measuring 25 $\mathrm{cm} \times 10 \mathrm{~cm}$. When attempting to harvest large flaps such as this, it is important to consider the number, distribution, and location of major perforators. In larger flaps, a relatively large perforator centered within the flap or multiple perforators may be advantageous. Vigilant monitoring of adequate venous drainage is also paramount. In addition, patients must be counseled about the need for a skin graft to close the donor site when large MSAP flaps are used. The MSAP flap has proven itself an effective and versatile option for a variety of defects across the body that would benefit from a thin fasciocutaneous flap. Moving forward, further research is warranted addressing the cutaneous territory supplied by the medial sural vessels as well as the use of the MSAP flap for trunk and pelvic reconstruction.

\section{NOTES}

\section{Conflict of interest}

No potential conflict of interest relevant to this article was reported. 


\section{Ethical approval}

The study was performed in accordance with the principles of the Declaration of Helsinki. Written informed consent was obtained.

\section{Patient consent}

The patient provided written informed consent for the publication and the use of his images.

\section{Author contribution}

Conceptualization: CM Teven, JW Yu, LC Zhao, JP Levine. Data curation: CM Teven, JW Yu, LC Zhao, JP Levine. Formal analysis: CM Teven, LC Zhao, JP Levine. Methodology: CM Teven, JW Yu, LC Zhao, JP Levine. Project administration: CM Teven, JP Levine. Visualization: CM Teven, JP Levine. Writing original draft: CM Teven, JW Yu, LC Zhao, JP Levine. Writing review \& editing: CM Teven, JW Yu, LC Zhao, JP Levine.

\section{ORCID}

Chad M. Teven https://orcid.org/0000-0002-2835-9942

\section{REFERENCES}

1. Taylor GI, Daniel RK. The anatomy of several free flap donor sites. Plast Reconstr Surg 1975;56:243-53.

2. Montegut W, Allen R. Sural artery perforator flap as an alternative for the gastrocnemius myocutaneous flap. Plast Reconstr Surg 1996;89:5113.

3. Cavadas PC, Sanz-Gimenez-Rico JR, Gutierrez-de la Camara A, et al. The medial sural artery perforator free flap. Plast Reconstr Surg 2001;108:1609-15.

4. Daar DA, Abdou SA, Cohen JM, et al. Is the medial sural artery perforator flap a new workhorse flap? A systematic review and meta-analysis. Plast Reconstr Surg 2019;143:393e403e.

5. Kao HK, Chang KP, Chen YA, et al. Anatomical basis and versatile application of the free medial sural artery perforator flap for head and neck reconstruction. Plast Reconstr Surg 2010;125:1135-45.

6. Ives M, Mathur B. Varied uses of the medial sural artery perforator flap. J Plast Reconstr Aesthet Surg 2015;68:853-8.

7. Hallock GG. Medial sural artery perforator free flap: legitimate use as a solution for the ipsilateral distal lower extremity defect. J Reconstr Microsurg 2014;30:187-92.

8. Hallock GG. A pragmatic evaluation of the role of the medial sural artery perforator flap. J Xiangya Med 2018;3:19.

9. Sano K, Hallock GG, Hamazaki M, et al. The perforatorbased conjoint (chimeric) medial Sural (MEDIAL GASTROCNEMIUS) free flap. Ann Plast Surg 2004;53:588-92.

10. Chiu T. Medial sural artery perforator flap. In: Van Hasselt A, Wong EWY; Chinese University of Hong Kong Faculty of Medicine, editors. Head \& neck dissection and reconstruction manual. Hong Kong: The Chinese University Press; 2005. p. 119-24.

11. Taylor GI, Pan WR. Angiosomes of the leg: anatomic study and clinical implications. Plast Reconstr Surg 1998;102: 599-616.

12. Taylor GI, Corlett RJ, Ashton MW. The functional angiosome: clinical implications of the anatomical concept. Plast Reconstr Surg 2017;140:721-33.

13. Wang X, Mei J, Pan J, et al. Reconstruction of distal limb defects with the free medial sural artery perforator flap. Plast Reconstr Surg 2013;131:95-105.

14. He Y, Jin SF, Zhang ZY, et al. A prospective study of medial sural artery perforator flap with computed tomographic angiography-aided design in tongue reconstruction. J Oral Maxillofac Surg 2014;72:2351-65.

15. Knott PD, Seth R, Waters HH, et al. Short-term donor site morbidity: a comparison of the anterolateral thigh and radial forearm fasciocutaneous free flaps. Head Neck 2016;38 Suppl 1:E945-8. 